\title{
ON CERTAIN HEATS OF DILUTION
}

\author{
BY J. E. TREVOR
}

\section{The Potential of a Component}

When the form of a Gibbsian 'fundamental equation' of a two-component system in a trivariant state, $i . e$. , in a one-phase state, is once found from experiment, the thermodynamic properties of the system in the state in question can be found by analytical means. The most convenient set of state variables to employ in the search for the form of a fundamental function of such a system is probably that composed of the pressure $p$, the absolute temperature $\theta$, and the masses $\mathrm{M}_{1}, \mathrm{M}_{2}$, of the independently variable components of the system. These are the variables of which the thermodynamic potential $\mathrm{H}$ of the system is a function. The thermodynamic potential $\mathrm{H}\left(p, \theta, \mathrm{M}_{1}, \mathrm{M}_{2}\right)$ can be found from the specific thermodynamic potential $h\left(p, \theta, m_{1}\right)$, where $m_{1}$ denotes the 'composition' of the phase,

$$
m_{1}=\frac{\mathrm{M}_{1}}{\mathrm{M}_{1}+\mathrm{M}_{2}}
$$

and the specific thermodynamic potential; because of the general relation

$$
h=m_{1} \mu_{1}+\left(\mathrm{I}-m_{1}\right) \mu_{2},
$$

can be found from the 'potentials' of the components, $\mu_{1}\left(p, \theta, m_{1}\right)$, $\mu_{2}\left(p, \theta, m_{1}\right)$; so the determination of the form of any fundamental function of the system can be reduced to the determination of the forms of these two potentials.

To the end of finding expressions for these potentials, suppose the mass $d \mathrm{M}_{j}$ of the $j$-th component to be added, irreversibly, to the mixture of mass

$$
\mathrm{M}_{1}+\mathrm{M}_{2}
$$

under the conditions of constant pressure, temperature, and mass of the other component. Before the addition, the energy of both masses is

$$
\mathrm{E}+e_{j} \cdot d \mathrm{M}_{j}
$$


where $\mathrm{E}$ is the energy $\mathrm{E}\left(p, \theta, \mathrm{M}_{1}, \mathrm{M}_{2}\right)$, of unknown form, of the system, and $e_{j}(p, \theta)$ is the specific energy of the $j$-th component. After the addition, the energy of the system is

$$
\mathrm{E}+\left\{e_{j}+\Delta_{j}-p\left(\frac{\partial \mathrm{V}}{\partial \mathrm{M}_{j}}-v_{j}\right)\right\} d \mathrm{M}_{j} ;
$$

where $\mathrm{V}\left(p, \theta, \mathrm{M}_{1}, \mathrm{M}_{2}\right)$ is the volume of the mixture, $v_{j}$ is the specific volume $v_{j}(p, \theta)$ of the $j$-th component, and $\Delta_{j}$ is the irreversible heat absorption accompanying the addition - i. e., $\Delta_{j}$ is the 'heat of dilution' by the $j$-th component.

In the addition, then, the energy of the mixture has increased by the amount

$$
\left\{e_{j}+\Delta_{j}-p\left(\frac{\partial \mathrm{V}}{\partial \mathrm{M}_{j}}-v_{j}\right)\right\} d \mathrm{M}_{j}=\frac{\partial \mathrm{E}}{\partial \mathrm{M}_{j}} d \mathrm{M}_{j},
$$

the rate of increase being equal to $\partial \mathrm{E} / \partial \mathrm{M}_{j}$, since it would have the same value were the addition in any way to be reversibly made. By changing variables in

$$
d \mathrm{E}=-p d \mathrm{~V}+\theta d \mathrm{H}+\mu_{1} d \mathrm{M}_{1}+\mu_{2} d \mathrm{M}_{2},
$$

where $H$ is the entropy of the system, we obtain for $\partial E / \partial M_{j}$ the general equation

$$
\frac{\partial \mathrm{E}}{\partial \mathrm{M}_{j}}=-p \frac{\partial \mathrm{V}}{\partial \mathrm{M}_{j}}+\theta \frac{\partial \mathrm{H}}{\partial \mathrm{M}_{j}}+\mu_{j}
$$

Elimination of $\partial \mathrm{E} / \partial \mathrm{M}_{j}$ between these two equations yields an expression for $\mu_{j}$,

$$
\mu_{j}=e_{j}+p v_{j}+\left(\Delta_{j}-\theta \frac{\partial \mathrm{H}}{\partial \mathrm{M}_{j}}\right) .
$$

In these equations, $e_{j}, v_{j}$ may be regarded as known functions of $p, \theta$; and $\Delta_{j}, \partial \mathrm{V} / \partial \mathrm{M}_{j}$ as experimentally determinable functions of $p, \theta, m_{1}$; but the function $\partial \mathrm{H}_{\partial} \partial \mathrm{M}_{1}$ of the variables $p, \theta, \mathrm{M}_{1}, \mathrm{M}_{2}$ remains of unknown form. In order to obtain a formulation of this function, we must consider the addition of the mass $a \mathrm{M}_{j}$ along a reversible path.

The reversible addition may be imagined to be effected by 
means of the arrangement described by Fig. I, which represents

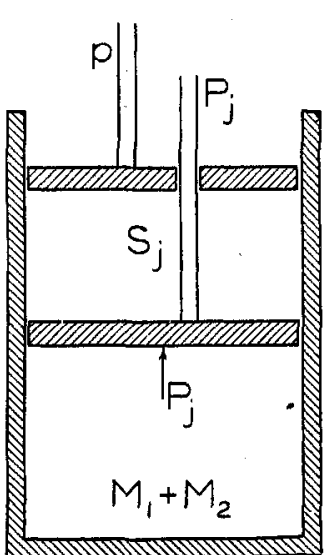

the mass $M_{1}+M_{2}$ enclosed in a cylinder and separated from a mass of the $j$-th com ponent $S_{j}$ by a piston permeable only by this component, the whole standing under the pressure $p$ exerted by an onter solid piston. The 'osmotic pressure' $\mathrm{P}_{j}$, with reference to the $\ddot{j}$-th component, of the system is balanced by a force applied from without to the semipermeable piston. An infinitesimal decrease of tihs force will result in a reversible addition of the mass $d \mathrm{M}_{j}$ to the mixture, at constant pressure, temperature,

Fig. I. and mass of the other component.

Before this reversible addition, the energy of the mass $\mathrm{M}_{1}+\mathrm{M}_{2}+d \mathrm{M}_{j}$ is

$$
\mathrm{E}+e_{j} \cdot d \mathrm{M}_{j,}
$$

after the addition it is

$$
\mathrm{E}+\left\{e_{j}-\mathrm{P}_{j} \frac{\partial \mathrm{V}}{\partial \mathrm{M}_{j}}-p\left(\frac{\partial \mathrm{V}}{\partial \mathrm{M}_{j}}-v_{j}\right)+\theta\left(\frac{\partial \mathrm{H}}{\partial \mathrm{M}_{j}}-\eta_{j}\right)\right\} d \mathrm{M}_{j}
$$

where $\eta_{j}$ is the specific entropy of the $j$-th component. The rate of increase of the energy of the mixture has therefore been

$$
e_{j}-\mathrm{P}_{j} \frac{\partial \mathrm{V}}{\partial \mathrm{M}_{j}}-p\left(\frac{\partial \mathrm{V}}{\partial \mathrm{M}_{j}}-v_{j}\right)+\theta\left(\frac{\partial \mathrm{H}}{\partial \mathrm{M}_{j}}-\eta_{j}\right)=\frac{\partial \mathrm{E}}{\partial \mathrm{M}_{j}} .
$$

We accordingly have :

In the irreversible addition,

$$
\frac{\partial \mathrm{E}}{\partial \mathrm{M}_{j}}=e_{j}-p\left(\frac{\partial \mathrm{V}}{\partial \mathrm{M}_{j}}-v_{j}\right)+\Delta_{j}
$$

in the reversible addition,

$$
\frac{\partial \mathrm{E}}{\partial \mathrm{M}_{j}}=e_{j}-p\left(\frac{\partial \mathrm{V}}{\partial \mathrm{M}_{j}}-v_{j}\right)+\theta\left(\frac{\partial \mathrm{H}}{\partial \mathrm{M}_{j}}-\eta_{j}\right)-\mathrm{P}_{j} \frac{\partial \mathrm{V}}{\partial \mathrm{M}_{j}} ;
$$


the general relation,

$$
\frac{\partial \mathrm{E}}{\partial \mathrm{M}_{j}}=\mu_{j}-p \frac{\partial \mathrm{V}}{\partial \mathrm{M}_{j}}+\theta \frac{\partial \mathrm{H}}{\partial \mathrm{M}_{j}} .
$$

Through elimination of common terms between these equations, we find :

From $(\alpha)$ and $(\beta)$,

$$
\Delta_{j}=\theta\left(\frac{\partial \mathrm{H}}{\partial \mathrm{M}_{j}}-\eta_{j}\right)-\mathrm{P}_{j} \frac{\partial \mathrm{V}}{\partial \mathrm{M}_{j}} ;
$$

From $(\alpha)$ and $(\gamma)$

$$
\mu_{j}=e_{j}+p v_{j}+\left(\Delta_{j}-\theta \frac{\partial \mathrm{H}}{\partial \mathrm{M}_{j}}\right)
$$

From $(\beta)$ and $(\gamma)$

$$
\mu_{j}=\left(e_{j}+p v_{j}-\theta \eta_{j}\right)-\mathrm{P}_{j} \frac{\partial \mathrm{V}}{\partial \mathrm{M}_{j}}
$$

The first of these equations determines $\partial \mathrm{H} / \partial \mathrm{M}_{j}$, whereupon the second determines $\mu_{j}$. The result of both together is given by $(7)$. Since the parenthesized expression in $(7)$ is the specific thermodynamic potential $h_{j}$ of the $j$-th component, this expression may be written

$$
\mu_{j}=h_{j}-\mathrm{P}_{j} \partial \mathrm{V} \mathrm{M}_{j} \quad(j=\mathrm{I}, 2)
$$

which is the relation sought. It may be noted here that, when the mass of the other component vanishes, $\mathrm{P}_{j}$ becomes zero and the equation reduces to

$$
\mu_{j}=h_{j}
$$

as it should.

\section{Another Derivation}

In place of successive reversible additions of the masses $d \mathrm{M}_{1}, d \mathrm{M}_{2}$ to the mixture having the mass $\mathrm{M}_{3}+\mathrm{M}_{2}$, let us 
imagine the described osmotic apparatus to be utilized for the reversible addition of the mass $M_{1}$ to the mass $M_{2}$, and for the addition of $M_{2}$ to $M_{1}$. In either case, the energy of both masses before the addition of $\mathrm{M}_{j}$ is

$$
e_{1} \mathrm{M}_{1}+e_{2} \mathrm{M}_{2}
$$

and after the addition it is

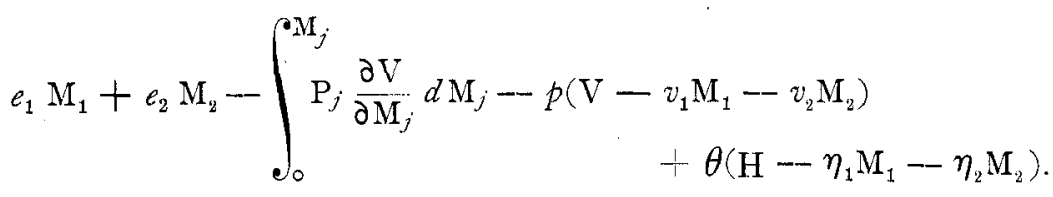

Equating the difference of these quantities with the change

$$
\mathrm{E}-e_{1} \mathrm{M}_{1}-e_{2} \mathrm{M}_{2}
$$

of the energy of the whole affair during the operation, and rearranging, we find

$$
\begin{aligned}
\mathrm{E}+p \mathrm{~V}-\theta \mathrm{H}=\mathrm{M}_{1}\left(e_{1}\right. & \left.+p v_{1}-\theta \eta_{1}\right) \\
& +\mathrm{M}_{2}\left(e_{2}+p v_{2}-\theta \eta_{2}\right)-\int_{0}^{\mathrm{M}_{j}} \mathrm{P}_{j} \frac{\partial \mathrm{V}}{\partial \mathrm{M}_{j}} d \mathrm{M}_{j} ;
\end{aligned}
$$

which may be written

$$
\mathrm{H}=\mathrm{M}_{1} h_{1}+\mathrm{M}_{2} h_{2}-\int_{0}^{\mathrm{M}_{j}} \mathrm{P}_{j} \frac{\partial \mathrm{V}}{\partial \mathrm{M}_{j}} d \mathrm{M}_{j}
$$

whence, on differentiation with regard to $\mathrm{M}_{j}$,

$$
\mu_{j}=h_{j}-\mathrm{P}_{j} \frac{\partial \mathrm{V}}{\partial \mathrm{M}_{j}^{\prime}}, \quad(j=\mathrm{I}, 2) \quad .
$$

as before. 


\section{The First Derivatives of the Thermodynamic Potential}

From the general relation

$$
\mathrm{V}=\left(\mathrm{M}_{1}+\mathrm{M}_{2}\right) \cdot v\left(p, \theta, m_{1}\right),
$$

where $v$ is the specific volume of the system, we find ${ }^{1}$

$$
\begin{aligned}
& \frac{\partial V}{\partial M_{1}}=v+\left(\mathrm{I}-m_{1}\right) \frac{\partial v}{\partial m_{1}}=\phi_{1}, \\
& \frac{\partial V}{\partial M_{2}}=v-m_{1} \cdot \frac{\partial v}{\partial m_{1}}=\phi_{2} .
\end{aligned}
$$

Incidentally it may be noted that $\phi_{1}, \phi_{2}$ are obvionsly equal to $\partial \mu_{1} / \partial p$ and $\partial \mu_{2} / \partial p$, respectively. The equations $\left(9^{a}\right),\left(9^{b}\right)$ convert the equations (8) for $\mu_{1}, \mu_{2}$ to

$$
\mu_{j}=h_{j}-\mathrm{P}_{j} \phi_{j} ; \quad(j=\mathrm{I}, 2)
$$

wherefore the general equation

$$
\mathrm{H}=\mathrm{M}_{1} \mu_{1}+\mathrm{M}_{2} \mu_{2}
$$

for the thermodynamic potential of the system in a trivariant state may be written

$$
\mathrm{H}=\mathrm{M}_{1}\left(h_{1}-\mathrm{P}_{1} \phi_{1}\right)+\mathrm{M}_{2}\left(h_{2}-\mathrm{P}_{2} \phi_{2}\right) .
$$

${ }^{1}$ If it should be desired to express $\phi_{1}, \phi_{2}$ by means of the specific volumes, or of the densities, of the components as they stand in the mixture, we should have

or

$$
\begin{gathered}
\phi_{1}=\left(1-m_{1}\right)^{2} \cdot \frac{\partial}{\partial m_{1}} \frac{v}{\mathrm{~s}-m_{1}} \\
\phi_{2}=-m_{1}^{2} \cdot \frac{\partial}{\partial m_{1}} \frac{v}{m_{1}}
\end{gathered}
$$

$$
\begin{aligned}
\phi_{1} & =-v^{2} \cdot \frac{\partial}{\partial m_{1}} \frac{1-m_{1}}{v} \\
\phi_{2} & =+v^{2} \cdot \frac{}{\partial m_{1}} \frac{m_{1}}{v} .
\end{aligned}
$$


Differentiating this equation,

$$
\begin{aligned}
d \mathrm{H} & =\left\{\mathrm{M}_{1}\left(v_{1}-\frac{\partial\left(\mathrm{P}_{1} \phi_{1}\right)}{\partial p}\right)+\mathrm{M}_{2}\left(v_{2}-\frac{\partial\left(\mathrm{P}_{2} \phi_{2}\right)}{\partial p}\right)\right\} d p \\
& -\left\{\mathrm{M}_{1}\left(\eta_{1}+\frac{\partial\left(\mathrm{P}_{1} \phi_{1}\right)}{\partial \theta}\right)+\mathrm{M}_{2}\left(\eta_{2}+\frac{\partial\left(\mathrm{P}_{2} \phi_{2}\right)}{\partial \theta}\right)\right\} d \theta \\
+ & \left\{h_{1}-\mathrm{P}_{1} \phi_{1}-\mathrm{M}_{1} \frac{\partial\left(\mathrm{P}_{1} \phi_{1}\right)}{\partial m_{1}} \frac{\partial m_{1}}{\partial \mathrm{M}_{1}}-\mathrm{M}_{2} \frac{\partial\left(\mathrm{P}_{2} \phi_{2}\right)}{\partial m_{1}} \frac{\partial m_{1}}{\partial \mathrm{M}_{1}}\right\} d \mathrm{M}_{1} \\
+ & \left\{h_{2}-\mathrm{P}_{2} \phi_{2}-\mathrm{M}_{2} \frac{\partial\left(\mathrm{P}_{2} \phi_{2}\right)}{\partial m_{1}} \frac{\partial m_{1}}{\partial \mathrm{M}_{2}}-\mathrm{M}_{1} \frac{\partial\left(\mathrm{P}_{1} \phi_{1}\right)}{\partial m_{1}} \frac{\partial m_{1}}{\partial \mathrm{M}_{2}}\right\} d \mathrm{M}_{2} ;
\end{aligned}
$$

whence we deduce

$$
\left\{\begin{array}{l}
\mathrm{V}=\mathrm{M}_{1}\left(v_{1}-\frac{\partial\left(\mathrm{P}_{1} \phi_{1}\right)}{\partial p}\right)+\mathrm{M}_{2}\left(v_{2}-\frac{\partial\left(\mathrm{P}_{2} \phi_{2}\right)}{\partial p}\right) \\
\mathrm{H}=\mathrm{M}_{1}\left(\eta_{1}+\frac{\partial\left(\mathrm{P}_{1} \phi_{1}\right)}{\partial \theta}\right)+\mathrm{M}_{2}\left(\eta_{2}+\frac{\partial\left(\mathrm{P}_{2} \phi_{2}\right)}{\partial \theta}\right)
\end{array}\right.
$$

or, dividing through by $\mathrm{M}_{1}+\mathrm{M}_{2}$,

$$
(\mathrm{I} 3 a)\left\{\begin{array}{l}
v=m_{1}\left(v_{1}-\frac{\partial\left(\mathrm{P}_{1} \phi_{1}\right)}{\partial p}\right)+\left(\mathrm{I}-m_{1}\right)\left(v_{2}-\frac{\partial\left(\mathrm{P}_{2} \phi_{2}\right)}{\partial p}\right) \\
\eta=m_{1}\left(\eta_{1}+\frac{\partial\left(\mathrm{P}_{1} \phi_{1}\right)}{\partial \theta}\right)+\left(\mathrm{I}-m_{1}\right)\left(\eta_{2}+\frac{\partial\left(\mathrm{P}_{2} \phi_{2}\right)}{\partial \theta}\right) .
\end{array}\right.
$$

where $\eta$ is the specific entropy of the system.

Rearranging these equations ( $3 \alpha$ ), and appending an equation obtained by comparing the equations $\mu_{j}=\partial \mathrm{H} / \partial \mathrm{M}_{j}$ with the initial equations for $\mu_{j}$, we find

(I4) $\left\{\begin{array}{l}m_{1} \frac{\partial\left(\mathrm{P}_{1} \phi_{1}\right)}{\partial p}+\left(\mathrm{I}-m_{1}\right) \frac{\partial\left(\mathrm{P}_{2} \phi_{2}\right)}{\partial p}=+m_{1} v_{1}+\left(\mathrm{I}-m_{1}\right) v_{2}-v \\ m_{1} \frac{\partial\left(\mathrm{P}_{1} \phi_{1}\right)}{\partial \theta}+\left(\mathrm{I}-m_{1}\right) \frac{\partial\left(\mathrm{P}_{2} \phi_{2}\right)}{\partial \theta}=-m_{1} \eta_{1}-\left(\mathrm{I}-m_{1}\right) \eta_{2}+\eta \\ m_{1} \frac{\partial\left(\mathrm{P}_{1} \phi_{1}\right)}{\partial m_{1}}+\left(\mathrm{I}-m_{1}\right) \frac{\partial\left(\mathrm{P}_{2} \phi_{2}\right)}{\partial m_{1}}=0 .\end{array}\right.$

These three equations may be regarded as expressing the relations between the slopes of the two osmotic pressure curves in the $\mathrm{P}, p$-, in the $\mathrm{P}, \theta$, and in the $\mathrm{P}, m_{1}$-diagram, at any assigned $p, \theta, m_{1}$. The second members of the first two of the 
equations are, respectively, the decrease of the specific volume and the increase of the specific entropy in the formation of the phase-mixture from its components. Denoting these changes of volume and entropy by $-\Delta v$ and $+\Delta \eta$, multiplying each equation through by the corresponding $d p, d \theta$, or $d m_{1}$, and adding, we find

$$
m_{1} \cdot d\left(\mathrm{P}_{1} \phi_{1}\right)+\left(\mathrm{I}-m_{1}\right) \cdot d\left(\mathrm{P}_{2} \phi_{2}\right)=-\Delta v \cdot d p+\Delta \eta \cdot d \theta .
$$

The first member of this equation is a portion of the differential of the increase $\Delta h$ of the thermodynamic potential in the formation of the phase-mixture from its components. We have, equation (II),

$$
\mathrm{H}=\mathrm{M}_{1}\left(h_{1}-\mathrm{P}_{1} \phi_{1}\right)+\mathrm{M}_{2}\left(h_{2}-\mathrm{P}_{2} \phi_{2}\right) ;
$$

whence, dividing through by $\mathrm{M}_{1}+\mathrm{M}_{2}$,

$$
\begin{aligned}
h & =m_{1}\left(h_{1}-\mathrm{P}_{1} \phi_{1}\right)+\left(\mathrm{I}-m_{1}\right)\left(h_{2}-\mathrm{P}_{2} \phi_{2}\right) \\
& =\left\{m_{1} h_{1}+\left(\mathrm{I}-m_{1}\right) h_{2}\right\}-\left\{m_{1} \mathrm{P}_{1} \phi_{1}+\left(\mathrm{I}-m_{1}\right) \mathrm{P}_{2} \phi_{2}\right\} ;
\end{aligned}
$$

whence

$$
\begin{aligned}
\Delta h & =-m_{1} \mathrm{P}_{1} \phi_{1}-\left(\mathrm{I}-m_{1}\right) \mathrm{P}_{2} \phi_{2}, \\
-d \Delta h & =m_{1} d\left(\mathrm{P}_{1} \phi_{1}\right)+\left(\mathrm{I}-m_{1}\right) d\left(\mathrm{P}_{2} \phi_{2}\right)+\left(\mathrm{P}_{1} \phi_{1}-\mathrm{P}_{2} \phi_{2}\right) d m_{1} ;
\end{aligned}
$$

wherefore, on comparison with (I5),

$$
-d \Delta h=-\Delta v \cdot d p+\Delta \eta \cdot d \theta+\left(\mathrm{P}_{1} \phi_{1}-\mathrm{P}_{2} \phi_{2}\right) \cdot d m_{1} .
$$

This equation (I6) corresponds to the general thermodynamic equation

$$
d h=v d p-\eta d \theta+\left(\mu_{1}-\mu_{2}\right) d m_{1} .
$$

The quantity $\Delta h$ is, of course, always negative. The equations

$$
\frac{\partial \Delta h}{\partial p}=\Delta v, \quad \frac{\partial \Delta h}{\partial \theta}=-\Delta \eta, \quad \frac{\partial \Delta h}{\partial m_{1}}=\mathrm{P}_{2} \phi_{2}-\mathrm{P}_{1} \phi_{1},
$$

are identical with the equations (I4), and so are equivalent to the 'derived relations'

$$
\frac{\partial \mathrm{H}}{\partial p}=\mathrm{V}, \quad \frac{\partial \mathrm{H}}{\partial \theta}=-\mathrm{H}, \quad \frac{\partial \mathrm{H}}{\partial m_{j}}=\mu_{j .} \quad(j=\mathrm{I}, 2)
$$

\section{Osmotic Pressures and Heats of Dilution}

The 'heats of dilution' $\Delta_{1}, \Delta_{2}$ of irreversible dilution of the 
phase-mixture are given by (5) and (6) as

$$
\begin{aligned}
& \Delta_{j}=-\mathrm{P}_{j} \frac{\partial \mathrm{V}}{\partial \mathrm{M}_{j}}+\theta\left(\frac{\partial \mathrm{H}}{\partial \mathrm{M}_{j}}-\eta_{j}\right) \\
& \Delta_{j}=\mu_{j}-e_{j}-p v_{j}+\theta \frac{\partial \mathrm{H}}{\partial \mathrm{M}_{j}} .
\end{aligned}
$$

If $\Delta_{j}$ be expressed with the aid of the osmotic pressures and the specific volume, the second of these formulations yields the same result as the first. We shall, therefore, employ the simpler of the two equations-the first.

As a preliminary, it is necessary to express $\partial \mathrm{H} / \partial \mathrm{M}_{j}$ with the aid of the osmotic pressures and the specific volume. We have, equation ( $\mathrm{I} 3$ ),

$$
\mathrm{H}=\mathrm{M}_{1}\left(\eta_{1}+\frac{\partial\left(\mathrm{P}_{1} \phi_{1}\right)}{\partial \theta}\right)+\mathrm{M}_{2}\left(\eta_{2}+\frac{\partial\left(\mathrm{P}_{2} \phi_{2}\right)}{\partial \theta}\right) ;
$$

whence

$$
\begin{gathered}
\frac{\partial \mathrm{H}}{\partial \mathrm{M}_{1}}=\eta_{1}+\frac{\partial\left(\mathrm{P}_{1} \phi_{1}\right)}{\partial \theta}+\mathrm{M}_{1} \frac{\partial^{2}\left(\mathrm{P}_{1} \phi_{1}\right)}{\partial \theta \partial m_{1}} \frac{\partial m_{1}}{\partial \mathrm{M}_{1}}+\mathrm{M}_{2} \frac{\partial^{2}\left(\mathrm{P}_{2} \phi_{2}\right)}{\partial \theta \partial m_{1}} \frac{\partial m_{1}}{\partial \mathrm{M}_{1}} \\
(\mathrm{I} 8 a)=\eta_{1}+\frac{\partial\left(\mathrm{P}_{1} \phi_{1}\right)}{\partial \theta}+m_{1}\left(\mathrm{I}-m_{1}\right) \frac{\partial^{2}\left(\mathrm{P}_{1} \phi_{1}\right)}{\partial \theta \partial m_{1}}+\left(\mathrm{I}-m_{1}\right)^{2} \frac{\partial^{2}\left(\mathrm{P}_{2} \phi_{2}\right)}{\partial \theta \partial m_{1}} \\
\frac{\partial \mathrm{H}}{\partial \mathrm{M}_{2}}=\eta_{2}+\frac{\partial\left(\mathrm{P}_{2} \phi_{2}\right)}{\partial \theta}+\mathrm{M}_{2} \frac{\partial^{2}\left(\mathrm{P}_{2} \phi_{2}\right)}{\partial \theta \partial m_{1}} \frac{\partial m_{1}}{\partial \mathrm{M}_{2}}+\mathrm{M}_{1} \frac{\partial^{2}\left(\mathrm{P}_{1} \phi_{1}\right)}{\partial \theta \partial m_{1}} \frac{\partial m_{1}}{\partial \mathrm{M}_{2}} \\
(\mathrm{I} 8 b)=\eta_{2}+\frac{\partial\left(\mathrm{P}_{2} \phi_{2}\right)}{\partial \theta}-m_{1}\left(\mathrm{I}-m_{1}\right) \frac{\partial^{2}\left(\mathrm{P}_{2} \phi_{2}\right)}{\partial \theta \partial m_{1}}-m_{1}{ }^{2} \frac{\partial^{2}\left(\mathrm{P}_{1} \phi_{1}\right)}{\partial \theta \partial m_{1}} .
\end{gathered}
$$

The last of equations (I4) convert (I $8 a)$ and (I $8 b$ ) to

$$
\left\{\begin{array}{l}
\partial \mathrm{H} \\
\partial \mathrm{M}_{1}=\eta_{1}+\frac{\partial\left(\mathrm{P}_{1} \phi_{1}\right)}{\partial \theta} \\
\frac{\partial \mathrm{H}}{\partial \mathrm{M}_{2}}=\eta_{2}+\frac{\partial\left(\mathrm{P}_{2} \phi_{2}\right)}{\partial \theta}
\end{array} ;\right.
$$

which convert (I7) to

$$
\begin{aligned}
& \Delta_{1}=\theta \frac{\partial\left(\mathrm{P}_{1} \phi_{1}\right)}{\partial \theta}-\mathrm{P}_{1} \phi_{1} \\
& \Delta_{2}=\theta \frac{\partial\left(\mathrm{P}_{2} \phi_{2}\right)}{\partial \theta}-\mathrm{P}_{2} \phi_{2} ;
\end{aligned}
$$


which equations may be written.

(2Ia)

$$
\Delta_{1}=\theta^{2} \cdot \frac{\partial}{\partial \theta} \frac{\mathrm{P}_{1} \phi_{1}}{\theta}, \quad \Delta_{2}=\theta^{2} \cdot \frac{\partial}{\partial \theta} \frac{\mathrm{P}_{2} \phi_{2}}{\theta} .
$$

It may be noted also that, because of equations (20), the two heats of dilution are connected by the relation

$$
\Delta_{2}-\Delta_{1}=\theta \cdot \frac{\partial}{\partial \theta}\left(\mathrm{P}_{2} \phi_{2}-\mathrm{P}_{1} \phi_{1}\right)-\left(\mathrm{P}_{2} \phi_{2}-\mathrm{P}_{1} \phi_{1}\right),
$$

which, since

$$
\mathrm{P}_{2} \phi_{2}-\mathrm{P}_{1} \phi_{1}=\frac{\partial \Delta h}{\partial m_{1}}
$$

may be written

$$
\begin{aligned}
\Delta_{2}-\Delta_{1} & =\frac{\partial}{\partial m_{1}}\left(\theta \frac{\partial \Delta h}{\partial \theta}-\Delta h\right) \\
& =\theta^{2} \cdot \frac{\partial}{\partial \theta \partial m_{1}} \frac{\Delta h}{\theta},
\end{aligned}
$$

a relation of simple and rather curious form.

The equations

$$
\mathrm{P}_{1}=\mathrm{P}_{1}\left(p, \theta, m_{1}\right), \quad \mathrm{P}_{2}=\mathrm{P}_{2}\left(p, \theta, m_{1}\right),
$$

for the two osmotic pressures of the phase-mixture are found from equations (2I)

$$
\frac{\partial}{\partial \theta} \frac{\mathrm{P}_{1} \phi_{1}}{\theta}=\frac{\Delta_{1}}{\theta^{2}}, \quad \frac{\partial}{\partial \theta} \frac{\mathrm{P}_{2} \phi_{2}}{\theta}=\frac{\Delta_{2}}{\theta^{2}}
$$

by integration with regard to $\theta$; which yields

$$
\mathrm{P}_{1} \phi_{1}=\theta \int \frac{\Delta_{1}}{\theta^{2}} d \theta, \quad \mathrm{P}_{2} \phi_{2}=\theta \int \frac{\Delta_{2}}{\theta^{2}} d \theta .
$$

Substituting for $\phi_{1}, \phi_{2}$ their values, as expressed by equations (9), we find

$$
\left\{\begin{aligned}
\mathrm{P}_{1}\left(v+\left(\mathrm{I}-m_{1}\right) \frac{\partial v}{\partial m_{1}}\right) & =\theta \int \frac{\Delta_{1}}{\theta^{2}} d \theta \\
\mathrm{P}_{2}\left(v-m_{1} \frac{\partial v}{\partial m_{1}}\right) & =\theta \int \frac{\Delta_{2}}{\theta^{2}} d \theta .
\end{aligned}\right.
$$


Evaluation of the second members, of these equations depends upon the formulation, from experiment, of the functions $\Delta,\left(p, \theta, m_{1}\right)$.

When the equations $(23 a)$,

$$
\begin{aligned}
& \mathrm{P}_{j} \phi_{j}=\theta \int \frac{\Delta_{j}}{\theta^{2}} d \theta \\
& =\theta \cdot \mathrm{D}_{\mathrm{i}}\left(p, \theta, m_{1}\right),
\end{aligned}
$$

representing the osmotic pressures by means of the heats of dilution, are combined with the equations (Io) for the component potentials,

$$
\mu_{i}=h_{j}-\mathrm{P}_{j} \phi_{j}
$$

and the resulting expressions for the potentials are substituted in the equation

$$
\mathrm{H}=\mathrm{M}_{\mathrm{i}} \mu_{1}+\mathrm{M}_{2} \mu_{2},
$$

for the thermodynamic potential of the phase-mixture, we obtain

$$
\mathrm{H}=\mathrm{M}_{1}\left(h_{1}-\theta \mathrm{D}_{1}\right)+\mathrm{M}_{2}\left(h_{2}-\theta \mathrm{D}_{2}\right) .
$$

From this equation it appears that appeal to experiment for the forms of the two functions $\Delta_{j}$ suffices for determination of the forms of the fundamental functions of a two-component phase-mixture, when the fundamental functions of the separate components are known. This is a very interesting general result.

Let us now return to consideration of the equations for the two osmotic pressures $\mathrm{P}_{j}$. If throughout any region of $p, \theta, m_{1^{-}}$ values it may be assumed that $\Delta_{1}=0$, or that $\Delta_{2}=0$, the equations (2I) become

$$
\frac{\partial}{\partial \theta} \frac{P_{1} \phi_{1}}{\theta}=0, \quad \frac{\partial}{\partial \theta} \frac{P_{2} \phi_{2}}{\theta}=0,
$$

whence

$$
\mathrm{P}_{1} \phi_{1}=r_{1}\left(p, m_{1}\right) \cdot \theta . \quad \mathrm{P}_{2} \phi_{2}=r_{2}\left(p, m_{2}\right) \cdot \theta .
$$

It thus appears that, when $\Delta_{j}=0$,

$$
\int \frac{\Delta_{j}}{\theta^{2}} d \theta=r_{j}
$$


When the phase-mixture is a 'dilute solution,' $m_{1}$ is small in comparison with unity, and $\partial v / \partial m_{1}$ is small in comparison with $v$, wherefore the first of equations (26) sensibly reduces to the van't Hoff equation,

$$
\mathrm{P}_{1} v=r_{1} \theta
$$

in which the function $r_{1}$, as was discovered by van 't Hoff from experimental evidence, is approximately equal to the ideal-gas constant $r$. The general form of the relation is, of course, that of the first equation $(23 b)$. Van 't Hoff's theory omits all consideration of the simultaneous value of the complementary osmotic pressure $\mathrm{P}_{2}$.

If the assumption of a vanishing heat of dilution may be replaced by the assumption that, in any region of $p, \theta, m_{1}$ values, $\Delta_{1}$ or $\Delta_{2}$ is independent of $\theta$, how will the osmotic pressures in this region depend upon the temperature? From the equations (20),

$$
\Delta_{j}=\theta \frac{\partial\left(\mathrm{P}_{j} \phi_{j}\right)}{\partial \theta}-\mathrm{P}_{\jmath} \phi_{j},
$$

the rates of change of the heats of dilution with changing temperature are found to be

$$
\frac{\partial \Delta_{j}}{\partial \theta}=\theta \frac{\partial^{2}\left(\mathrm{P}_{j} \phi_{j}\right)}{\partial \theta^{2}}
$$

wherefore, in the region in question,

$$
\begin{array}{r}
\frac{\partial^{2}\left(\mathrm{P}_{j} \phi_{j}\right)}{\partial \theta^{2}}=0, \\
\frac{\partial\left(\mathrm{P}_{j} \phi_{j}\right)}{\partial \theta}=f_{j}, \\
\mathrm{P}_{j} \phi_{j}=f_{j} \theta+g_{j},
\end{array}
$$

where the $J$ 's and $g$ 's are independent of $\theta$. These equations differ in form from the equations (26) by an additive term.

$$
\text { The Locus } P_{1}=P_{2}
$$

Let the plane diagram of Fig. 2 represent the osmotic pressures $\mathrm{P}_{1}, \mathrm{P}_{2}$ of a phase-mixture, in their dependence on the com- 
position $m_{1}$ at any assigned values of $p, \theta$. Since both curves rise from zero values, they will intersect at a point $a$. The equation $\mathrm{P}_{1}=\mathrm{P}_{2}$ is the locus $\mathrm{R}\left(p, \theta, m_{1}\right)=0$ of the points $a$.

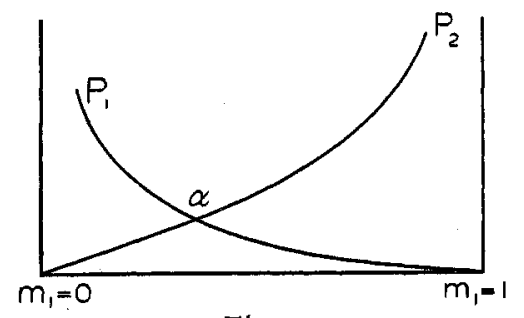

Fig. 2

If the variables $p, \theta, m_{1}$ be represented by rectangular coordinates in space, to every point of the space in which the variables have possible values will correspond a pair of values of $\mathrm{P}_{1}, \mathrm{P}_{2}$; and the equation $\mathrm{R}=\mathrm{O}$ will represent a surface that is the locus of the points at which $\mathrm{P}_{1}, \mathrm{P}_{2}$ are equal. Let us seek to connect the common value $\mathrm{P}_{\circ}$ of the osmotic pressures at this locus with the specific thermodynamic potential $h\left(p, \theta, m_{1}\right)$ of the system.

A general thermodynamic relation that will be of service in this task is

$$
\begin{aligned}
\frac{\partial V}{\partial \mathrm{M}_{\mathcal{J}}} & =\frac{\partial}{\partial p} \mu_{j}\left(p, \theta, \mathrm{M}_{1}, \mathrm{M}_{2}\right), \\
& =\frac{\partial}{\partial p} \mu_{i}\left(p, \theta, m_{1}\right),
\end{aligned}
$$

which is one of the conditions of integrability of the differential of the thermodynamic potential $\mathrm{H}$,

$$
\mathrm{V} d p-\mathrm{H} d \theta+\mu_{1} d \mathrm{M}_{1}+\mu_{2} d \mathrm{M}_{2} .
$$

Further requisite general relations are obtained from

$$
\mathrm{H}=\left(\mathrm{M}_{1}+\mathrm{M}_{2}\right) \cdot h \text {, }
$$

whence follows

$$
\frac{\partial \mathrm{H}}{\partial \mathrm{M}_{j}}=h+\left(\mathbf{M}_{1}+\mathbf{M}_{2}\right) \frac{\partial h \partial m_{1}}{\partial m_{1} \partial \mathbf{M}_{j}}
$$


wherefore

$$
\left\{\begin{array}{l}
\mu_{1}=h+\left(1-m_{1}\right) \frac{\partial h}{\partial m_{1}}, \\
\mu_{2}=h-m_{1} \frac{\partial h}{\partial m_{1}},
\end{array}\right.
$$

which are the equations in question.

Now, from the equations (8)

$$
\mathrm{P}_{j}=\frac{h_{j}-\mu_{j}}{\frac{\partial \mathrm{V}}{\partial \mathrm{M}_{j}}}
$$

the equation $\mathrm{P}_{1}=\mathrm{P}_{\mathrm{z}}$ of the loctis may be written

$$
\left(h_{1}-\mu_{1}\right) \frac{\partial \mathrm{V}}{\partial \mathrm{M}_{2}}=\left(h_{2}-\mu_{2}\right) \frac{\partial \mathrm{V}}{\partial \mathrm{M}_{1}},
$$

which is converted by $(a)$ and $(b)$ to

$$
\left(h_{2}-h_{1}+\frac{\partial h}{\partial m_{1}}\right) \frac{\partial h}{\partial p}=\left(h-m_{1} h_{1}-\left(\mathrm{I}-m_{1}\right) h_{2}\right) \frac{\partial^{2} h}{\partial \partial \partial m_{1}},
$$

which, because of

$$
h-m_{1} h_{1}-\left(\mathrm{I}-m_{1}\right) h_{2}=\Delta h,
$$

becomes

$$
\begin{array}{c|c|}
\Delta h & \frac{\partial h}{\partial p} \\
\frac{\partial \Delta h}{\partial m_{1}} & \frac{\partial^{2} h}{\partial p \partial m_{1}}
\end{array} \mid=0,
$$

which is the equation of the locus. The vanishing of this Wronskian of $\Delta h$ and $\partial h / \partial p$ requires that these functions shall be connected by a linear relation

$$
-\Delta h=z(p, \theta) \cdot \frac{\partial h}{\partial p} .
$$

We shall shortly see that the coefficient $z$ of this relation is equal to the common value $P_{0}$ of the two osmotic pressures. 
Let 11s now seek to express $P_{0}$. From (I6) we have

$$
\frac{\partial \Delta h}{\partial m_{1}}=\mathrm{P}_{2} \phi_{2}-\mathrm{P}_{1} \phi_{1}
$$

which equation connecting $\mathrm{P}_{1}, \mathrm{P}_{2}$ becomes, for $\mathrm{P}_{1}=\mathrm{P}_{2}=\mathrm{P}_{\mathrm{o}}$,

$$
\mathrm{P}_{\circ}\left(\phi_{2}-\phi_{1}\right)=\frac{\partial \Delta h}{\partial m_{1}}
$$

Now, by $(a)$ and $(b)$,

$$
\begin{aligned}
& \phi_{2}=\frac{\partial V}{\partial M_{2}}=\frac{\partial \mu_{2}}{\partial p}=\frac{\partial h}{\partial p}-m_{1} \frac{\partial^{2} h}{\partial p \partial m_{1}}, \\
& \phi_{1}=\frac{\partial V}{\partial M_{1}}=\frac{\partial \mu_{1}}{\partial p}=\frac{\partial h}{\partial p}+\left(1-m_{1}\right) \frac{\partial^{2} h}{\partial p \partial m},
\end{aligned}
$$

whence

$$
\phi_{2}-\phi_{1}=-\frac{\partial^{2} h}{\partial \not \partial m_{1}}
$$

which converts the above equation for $P_{0}$ to

$$
\mathrm{P}_{\circ}=-\frac{\partial \Delta h}{\partial m_{1}}: \frac{\partial^{2} h}{\partial p \partial m_{1}},
$$

the equation for the osmotic pressure at the locus. Elimination of

$$
\frac{\partial \Delta h}{\partial m_{1}}: \frac{\partial^{2} h}{\partial p \partial m_{1}}
$$

between (28) and (29) yields a second equation for $P_{0}$,

$$
-\mathrm{P}_{\circ}=\Delta h: \frac{\partial h}{\partial p}
$$

Starting with the equation (29) for $\mathrm{P}_{0}$, we can obtain a succession of expressions for the locus $\mathrm{P}_{1}==\mathrm{P}_{2}$, and also for the osmotic pressure there. Since the second member of this equation must be a function of $p, \theta$ only, we have, equating its $m_{1}$ derivative to zero,

$$
\left(\frac{\partial^{2} h}{\partial p \partial m_{1}} \frac{\partial^{2} \Delta h}{\partial m_{1}^{2}}-\frac{\partial \Delta h}{\partial m_{1}} \frac{\partial^{3} h}{\partial p \partial m_{1}^{2}}\right):\left(\frac{\partial^{2} h}{\partial p \partial m_{1}}\right)^{2}=0 .
$$


The denominator of the fraction here, which is equal to $\left(\partial v / \partial m_{1}\right)^{2}$, presumably does not vanish ; so we have

$$
\left|\begin{array}{lc}
\frac{\partial \Delta h}{\partial m_{1}} & \frac{\partial^{2} h}{\partial p \partial m_{1}} \\
\frac{\partial^{2} \Delta h_{4}}{\partial m_{1}^{2}} & \frac{\partial^{3} h}{\partial p \partial m_{1}^{2}}
\end{array}\right|=0 .
$$

Eliminating

$$
\frac{\partial \Delta h}{\partial m_{1}}: \frac{\partial^{2} h}{\partial p \partial m_{1}}
$$

between (3I) and (29), and noting that $\partial^{2} \Delta h / \partial m_{1}^{2}=\partial^{2} h / \partial m_{1}^{2}$, we find a corresponding third expression for the osmotic pressure at the locus,

$$
\mathrm{P}_{\circ}=-\frac{\partial^{2} h}{\partial m_{1}^{2}}: \frac{\partial^{8} h}{\partial p \partial m_{1}^{2}}
$$

The second member of (32) is, in its turn, a function of $p, \theta$; wherefore we can proceed as above, obtaining another pair of equations analogons to (3I) and (32), and continue the process ad infinitum.

We thus have, collecting the equations (28) to (32), the successive equations

$$
\text { (33) } \quad \mathrm{P}_{\circ}=-\frac{\Delta h}{\frac{\partial h}{\partial p}}=-\frac{\frac{\partial \Delta h}{\partial m_{1}}}{\frac{\partial^{2} h}{\partial p \partial m_{1}}}=-\frac{\frac{\partial^{2} h}{\partial m_{1}^{2}}}{\frac{\partial^{3} h}{\partial p \partial m_{1}^{2}}}=\ldots
$$

and the zero Wronskian matrix

$$
\left|\begin{array}{cc}
\Delta h & \frac{\partial h}{\partial p} \\
\frac{\partial \Delta h}{\partial m_{1}} & \frac{\partial^{2} h}{\partial p \partial m_{1}} \\
\frac{\partial^{2} h}{\partial m_{1}{ }^{2}} & \frac{\partial^{3} h}{\partial p \partial m_{1}{ }^{2}} \\
\ldots & \ldots
\end{array}\right|=0 .
$$


The vanishing of this matrix, being the condition that the functions constituting each of its rows shall be connected by a linear relation whose coefficient is independent of $m_{1}$, is the condition for the existence of the equations (33). When the first column is replaced by the values derived from the first of equations (33)

$$
\Delta h=-\mathrm{P}_{\circ} \partial \frac{\partial h}{\partial p}
$$

the matrix becomes

$$
\left|\begin{array}{cc}
-\mathrm{P}_{\circ} \frac{\partial h}{\partial p} & \frac{\partial h}{\partial p} \\
-\mathrm{P}_{\circ} \frac{\partial^{2} h}{\partial p \partial m_{1}} & \frac{\partial^{2} h}{\partial p \partial m_{1}} \\
-\mathrm{P}_{\circ} \frac{\partial^{3} h}{\partial p \partial m_{1}{ }^{2}} & \frac{\partial^{3} h}{\partial p \partial m_{1}{ }^{2}} \\
\ldots & \ldots
\end{array}\right|=0,
$$

which is obviously identically satisfied. It is, finally, not without interest to note that the equations (33) may be given the forms

$$
-\Delta h=\mathrm{P}_{\circ} v, \quad-\frac{\partial \Delta h}{\partial m_{1}}=\mathrm{P}_{\circ} \frac{\partial v}{\partial m_{1}}, \quad \text { etc. }
$$

From this equation we may obtain some curious information concerning the form of the thermodynamic potential, and the forms of the component-potentials, of the phase-mixture. From the equation

$$
\Delta h=h-m_{1} h_{1}-\left(\mathrm{I}-m_{1}\right) h_{2},
$$

we find

$$
h=\Delta^{h}+\left(h_{1}-h_{2}\right) m_{1}+h_{2} ;
$$

whence, from (33), at the locus,*

$$
h=-\mathrm{P}_{\mathrm{o}} \partial \hat{p}+\left(h_{1}-h_{2}\right) m_{1}+h_{2} .
$$

* Note that this equation is

$$
h=-\mathrm{P}_{\circ} v+\left(h_{1}-h_{2}\right) m_{1}+h_{2} .
$$


Further, from the general equations $(b)$,

$$
\begin{gathered}
\mu_{1}=h+\left(\mathrm{I}-m_{1}\right) \frac{\partial h}{\partial m_{1}}, \\
\mu_{2}=h-m_{1} \frac{\partial h}{\partial m_{1}},
\end{gathered}
$$

substituting for $h$ from (35)

$$
\begin{aligned}
& \mu_{1}=-\mathrm{P}_{\circ}\left(\frac{\partial h}{\partial p}+\left(\mathrm{I}-m_{1}\right) \frac{\partial^{2} h}{\partial p \partial m_{1}}\right)+h_{1}, \\
& \mu_{2}=-\mathrm{P}_{\circ} \cdot\left(\frac{\partial h}{\partial p}-m_{1} \frac{\partial^{2} h}{\partial p \partial m_{1}}\right)+h_{2},
\end{aligned}
$$

and replacing $\partial h / \partial p$ by the specific volume $v$ of the mixture, we find that, at the locus,

$$
\begin{aligned}
& \mu_{1}-h_{1}=\mathrm{P}_{\circ} v^{2} \cdot \frac{\partial}{\partial m_{1}} \frac{\mathrm{I}-m_{1}}{v} \\
& \mu_{2}-h_{2}=-\mathrm{P}_{\circ} v^{2} \cdot \frac{\partial}{\partial m_{1}} \frac{m_{1}}{v} .
\end{aligned}
$$

The quantities whose composition-derivatives appear in these equations are successively the densities, in the mixture, of the first and of the second components.

\section{Formulation of Heats of Dilution}

In conclusion, let us now formulate the heats of dilution $\Delta_{1}, \Delta_{2}$, of irreversible dilution of a two-component phase-mixture, by means of the specific thermodynamic potential of the mixture. For these heats of dilution we have the equations (20),

$$
\Delta_{1}=\theta \frac{\partial\left(\mathrm{P}_{1} \phi_{1}\right)}{\partial \theta}-\mathrm{P}_{1} \phi_{1} . \quad \Delta_{2}=\theta \frac{\partial\left(\mathrm{P}_{2} \phi_{2}\right)}{\partial \theta}-\mathrm{P}_{2} \phi_{2} .
$$

By equations (Io) and (b) we have, respectively,

$$
\begin{array}{cl}
\mathrm{P}_{1} \phi_{1}=h_{1}-\mu_{1}, & \mathrm{P}_{2} \phi_{2}=h_{2}-\mu_{2}, \\
\mu_{1}=h+\left(\mathrm{I}-m_{1}\right) \frac{\partial h}{\partial m_{1}}, & \mu_{2}=h-m_{1} \frac{\partial h}{\partial m_{1},}
\end{array}
$$

wherefore

$$
\mathrm{P}_{1} \phi_{1}=h_{1}-h-\left(\mathrm{I}-m_{1}\right) \frac{\partial h}{\partial m_{1}} ; \quad \mathrm{P}_{2} \phi_{2}=h_{2}-h+m_{1} \frac{\partial h}{\partial m_{1}} ;
$$


which values serve to convert the above equations for $\Delta_{1}, \Delta_{2}$ to (37a) $\Delta_{1}==-\left(h_{1}-\theta \frac{\partial h_{1}}{\partial \theta}\right)+\left(h-\theta \frac{\partial h}{\partial \theta}\right)+$

$$
\left(1-m_{1}\right) \cdot \frac{\partial}{\partial m_{1}}\left(h-\theta \frac{\partial h}{\partial \theta}\right)
$$

$(37 b) \Delta_{2}=-\left(h_{2}-\theta \frac{\partial h_{2}}{\partial \theta}\right)+\left(h-\theta \frac{\partial h}{\partial \theta}\right)-m_{1} \cdot \frac{\partial}{\partial m_{1}}\left(h-\theta \frac{\partial h}{\partial \theta}\right)$.

The expression $h-\theta \cdot \partial h \partial \partial$ is the specific Gibbsian heatfunction, defined by the equation

$$
g=e+p v
$$

wherefore the equations (37) may be expressed in the compact form

$$
\Delta_{1}=\left(g-g_{1}\right)+\left(\mathrm{I}-m_{1}\right) \frac{\partial g}{\partial m_{1}}
$$

$$
\Delta_{2}=\left(g-g_{2}\right)-m_{1} \frac{\partial g}{\partial m_{1}} \text {. }
$$

Expressing the second member of each of these equations by means of a derivative of a single function, we find

$$
\begin{gathered}
\Delta_{1}=\left(\mathrm{I}-m_{1}\right)^{2} \cdot \frac{\partial}{\partial m_{1}} \frac{g-g_{1}}{\mathrm{I}-m_{1}} \\
\Delta_{2}=-m_{1}^{2} \cdot \frac{\partial}{\partial m_{1}} \frac{g-g_{2}}{m_{1}} .
\end{gathered}
$$

Finally, by subtraction, the equations $(38)$ yield

$$
\Delta_{1}-\Delta_{2}=g_{2}-g_{1}+\frac{\partial g}{\partial m_{1}}
$$

$$
=\frac{\partial \Delta g}{\partial m_{1}}
$$

when $\Delta g$ is written for the increase

$$
g-m_{1} g_{1}-\left(i-m_{1}\right) g_{2}
$$

of the heat-function in the formation of unit mass of the phasemixture from its components. 
If the formulas for the heats of dilution be assembled, for comparison, we shall have:

$$
\begin{aligned}
& (2 \mathrm{I} a) \quad\left\{\begin{aligned}
\Delta_{1} & =\theta^{2} \cdot \partial \frac{\partial}{\partial \theta} \frac{\mathrm{P}_{1} \phi_{1}}{\theta} \\
\Delta_{2} & =\theta^{2} \cdot \frac{\partial}{\partial \theta} \frac{\mathrm{P}_{2} \phi_{2}}{\theta} \\
\Delta_{1}-\Delta_{2} & :=\theta^{2} \cdot \frac{\partial}{\partial \theta} \frac{\mathrm{P}_{1} \phi_{1}}{\theta}-\mathrm{P}_{2} \phi_{2}
\end{aligned}\right. \\
& (37 \alpha) \quad \Delta_{1}=\left(\mathrm{I}-m_{1}\right)^{2} \cdot \frac{\partial}{\partial m_{1}} \frac{\left(h-\theta \frac{\partial h}{\partial \theta}\right)-\left(h_{1}-\theta \frac{\partial h_{1}}{\partial \theta}\right)}{\mathrm{I}-m_{1}} \\
& (376)\left\{\Delta_{2}=-m_{1}{ }^{2} \cdot \partial m_{1} \frac{\left(h-\theta \frac{\partial h}{\partial \theta}\right)-\left(h_{2}-\theta \frac{\partial h_{2}}{\partial \theta}\right)}{m_{1}}\right. \\
& \text { (22) } \mid \Delta_{1}-\Delta_{2}=-\theta^{2} \cdot \partial \theta \partial \partial_{1}^{2} \frac{\Delta h}{\theta} \\
& (39 a) \\
& \Delta_{1}=\left(\mathrm{I}-m_{1}\right)^{2} \cdot \frac{\partial}{\partial m_{1}} \mathrm{~g}-g_{1} \\
& \Delta_{2}=\quad-m_{1}^{2} \cdot \frac{\partial}{\partial m_{1}} \frac{g-g_{2}}{m_{1}} \\
& \text { (40) } \quad \mid \begin{array}{l}
\frac{\partial \Delta g}{\partial m_{1}} \\
\Delta_{1}-\Delta_{2}=
\end{array}
\end{aligned}
$$

Cornell University. November, Igo4. 